\title{
Efectos de los Active Flux sobre Soldaduras GMAW Aplicadas a un Acero AISI/SAE 1020
}

\author{
Cristhian Harley Madariaga Rosas ${ }^{1}$ (D) , Paulo José Modenesi², Mauricio Rincón Ortiz ${ }^{1}$ (D) \\ ${ }^{1}$ Universidad Industrial de Santander - UIS, Escuela de Ingeniería Metalúrgica y Ciencia de Materiales, Bucaramanga, Santander, Colombia. \\ 2 Universidade Federal de Minas Gerais - UFMG, Departamento de Engenharia Metalúrgica e de Materiais, Belo Horizonte, MG, Brasil.
}

Como citá: Madariaga Rosas $\mathrm{CH}$, Modenesi PJ, Rincón Ortiz M. Efectos de los active flux sobre soldaduras GMAW aplicadas a un acero AISI/SAE 1020. Soldagem \& Inspeção. 2020;25:e2502. https://doi.org/10.1590/0104-9224/SI25.02

\begin{abstract}
Resumen: GMAW es uno de los procesos de soldadura más utilizados en la industria por su fácil utilización y automatización, lo que le da un excelente nivel de productividad y versatilidad. Una variante de este proceso, aún poco estudiada, es el A-GMAW (Active flux Gas Metal Arc Welding), que consiste en la adición de una fina capa de óxidos o haluros en polvo. El presente trabajo consistió en una evaluación de los efectos causados por la aplicación de flux de $\mathrm{CaCO}_{3}$ y $\mathrm{SiO}_{2}$ sobre la morfología del cordón, los parámetros operacionales y la estabilidad del proceso, en unas soldaduras GMAW aplicadas sobre un acero AISI/SAE 1020. Para esto se utilizó una fuente de soldadura operada en modo corriente constante y se hicieron ensayos pre-liminares donde se determinaron los valores de los parámetros a utilizar, los cuales se mantuvieron constantes durante todo el transcurso del trabajo. Los resultados obtenidos demostraron que los flux de $\mathrm{CaCO}_{3}$ y $\mathrm{SiO}_{2}$, sí tienen influencia en cambios morfológicos, como la penetración de la soldadura, e igualmente se observaron alteraciones en los modos de transferencia que gobernaban cada una de las soldaduras, así como en su estabilidad.
\end{abstract}

Palabras clave: A-GMAW; Active flux; Tensión; Soldadura; Transferencia metálica.

\section{Effects of Active Flux on GMAW Welding Applied to a Steel AISI/SAE 1020}

\begin{abstract}
GMAW is one of the most used welding processes in the industry. It is easy to use and to automate, and these characteristics allow high productivity and versatility. Currently, a process variant known as "A-GMAW" (Active flux Gas Metal Arc Welding) has a characteristic that has not been widely studied, it consists on the addition of a thin layer of oxides or halides in powder on the base metal surface. This research aims to evaluate the effects caused by the application of " $\mathrm{CaCO}_{3}$ and $\mathrm{SiO}_{2}$ fluxes on the morphology of the weld bead, operating parameters and process stability. This analysis was carried out in GMAW welding applied on an AISI/SAE 1020 steel. The results show the significant effects of $\mathrm{CaCO}_{3}$ and $\mathrm{SiO}_{2}$ fluxes. These additives influence the morphology, such as the penetration of the weld, and changes were also observed in the transfer modes that governed each of the welds, likewise the stability of the process also presents alterations.
\end{abstract}

Keywords: A-GMAW; Flux active; Voltage; Welding; Metal transfer.

\section{Introducción}

GMAW (del inglés Gas Metal Arc Welding) o MIG/MAG (del inglés Metal Inert Gas/Metal Active Gas) es uno de los procesos de soldadura más utilizados en la industria por sus grandes ventajas en comparación a otros procesos, debido a su alimentación continua y mayores tasas de deposición, lo cual deriva a una mayor productividad. Dentro del proceso GMAW se distinguen diferentes mecanismos de transferencia entre los cuales se encuentran el cortocircuito, globular y rocío (spray), donde estos dos últimos tienen la característica que la gota que se deprende del electrodo viaja por vuelo libre hacia el charco de soldadura. Dichos mecanismos de transferencia dependen de diferentes parámetros y características de proceso, de los cuales se destaca principalmente la corriente aplicada, la cual va determinar el tamaño y el número de gotas transferidas del electrodo hacia el charco de soldadura [1-3]. Sin embargo, debido al elevado número de variables interrelacionadas que involucra el proceso de soldadura, muchas de estas son seleccionadas de una forma empírica con base en experiencia práctica y estudios previos [4]. De este modo, un buen ajuste y control de los parámetros es vital para el éxito del proceso, pues según su ajuste, puede dar como resultado diferentes mecanismos de transferencia metálica, que están relacionados con el rendimiento en que se deposita la gota de metal en el charco de fusión, de no ser así puede generar inestabilidad operacional del arco y por ende, una morfología del cordón de soldadura defectuosa o no deseada [2]. En los últimos años, se han venido desarrollando fuentes de energía con sistemas de control eléctrico y/o mecánicos, para el control y monitoreo de los parámetros; pero la implementación de estas tecnologías eleva los costos de producción, además de requerir un mantenimiento más especializado, y ser más complejas al momento de operarlas [5]. Por eso, hoy en día se abren líneas de investigación que buscan un mayor control de los resultados 
finales; a través, de la utilización de active flux en la soldadura GMAW. Esta variante del proceso es conocida como A-GMAW y consiste en la adición de una fina capa de óxidos o halógenos sobre el metal base a soldar. No obstante, es una aplicación nueva y poco explorada para este tipo de soldadura, pues sus bases radican en el proceso A-TIG (variante del proceso GTAW), en donde las investigaciones han sido mayores y se han encontrado efectos repetitivos en cambios morfológicos del cordón de soldadura, tanto en apariencia, como en ancho y penetración, alcanzado aumentos de casi $100 \%$ en esta última característica [6-8]. En los escasos estudios realizados con A-GMAW, los autores han observado que los flux generan modificaciones en las variables operacionales, que terminan en alteraciones del periodo y frecuencia de la transferencia metálica, así como en cambios morfológicos del cordón [6,9].

De acuerdo a lo expuesto, la implementación de los active flux en soldaduras GMAW se convierte en una alternativa para modificar las características operacionales del proceso, de una forma menos compleja, y esto permite definir el objetivo propuesto del presente trabajo, que consistió en evaluar los efectos generados por los active flux en el proceso de soldadura GMAW de un acero AISI/SAE 1020.

\section{Metodología Experimental}

La realización de esta investigación condujo a varias etapas, que consistieron en la caracterización y análisis granulométrico de los flux a utilizar, se prepararon las probetas y se realizaron los ensayos de soldadura, con su respectiva adquisición de señales eléctricas con las que se analizó el comportamiento de las variables operacionales del proceso, y posteriormente se realizó el corte de dichas probetas soldadas, para finalmente realizar la preparación y análisis metalográfico.

EI material de las probetas utilizadas fue un acero al carbono AISI/SAE 1020, que está clasificado como un acero de bajo carbono y todas éstas tenían unas mismas dimensiones (Figura 1). Para este proceso fueron establecidos unos parámetros con los que se realizaron todas las soldaduras, lo mismo que los valores de corriente con los que se hicieron las soldaduras con active flux. Dichos parámetros fueron obtenidos mediante un primer ensayo del proceso GMAW, haciéndose un barrido desde 150 Amperios $[\mathrm{A}]$ y aumentando de $10 \mathrm{~A}$, hasta llegar a 250A. En respuesta a estas variables mencionadas anteriormente, el equipo operó con los valores de velocidad de alimentación y tensión necesarios. (Ver parámetros en la Tabla 1).

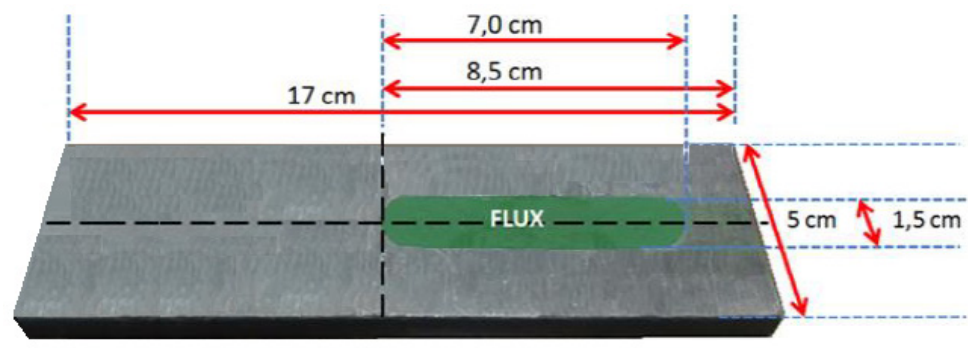

Figura 1. Dimensiones de la chapa metálica utilizada [9].

El carbonato de calcio $\left(\mathrm{CaCO}_{3}\right)$ y el óxido de silicio $\left(\mathrm{SiO}_{2}\right)$ empleados como active flux tuvieron una granulometría ajustada (< malla 200). Los anteriores flux se escogieron por no ser higroscópicos y por ser compuestos generalmente usados en la fabricación de los electrodos revestidos [9], así como por resultados preliminares obtenidos de un proceso A-GMAW con transferencia por corto-circuito. Para la deposición del flux en los cuerpos de prueba, se utilizó como referencia lo realizado en otros estudios similares $[9,10]$. Se delimitó la zona donde iría depositado el flux, dejando un área de $1000 \mathrm{~mm}^{2}$, sobre la cual se depositarían dichos fundentes. La cantidad de flux correspondía a 0,1 g para cada prueba, el cual daba como resultado una densidad de $100 \mathrm{~g} / \mathrm{m}^{2}$ de flux en el área delimitada.

Tabla 1. Parámetros fijos de los procesos de soldadura.

\begin{tabular}{|c|c|c|}
\hline \multirow{2}{*}{ PARÁMETRO } & \multicolumn{2}{|c|}{ CONFIGURACIÓN } \\
\hline & Mínimo & Máximo \\
\hline Corriente transferencia globular & $190 \mathrm{~A}$ & $210 \mathrm{~A}$ \\
\hline Corriente transferencia spray & \multicolumn{2}{|c|}{$230 \mathrm{~A}$} \\
\hline Modo de operación de la fuente y tipo de corriente & \multicolumn{2}{|c|}{ Corriente continua $(\mathrm{CC}+)$} \\
\hline Velocidad de soldadura & \multicolumn{2}{|c|}{$40[\mathrm{~cm} / \mathrm{min}]$} \\
\hline Electrodo (alambre) & \multicolumn{2}{|c|}{ ER70S6-1,2 [mm] } \\
\hline Gas de protección / Caudal & \multicolumn{2}{|c|}{$\mathrm{Ar}-4 \% \mathrm{CO}_{2} / 19[\mathrm{~L} / \mathrm{min}]$} \\
\hline Longitud del arco en la región sin flux & \multicolumn{2}{|c|}{$6[\mathrm{~mm}]$} \\
\hline Orientación del electrodo & \multicolumn{2}{|c|}{ Perpendicular } \\
\hline Distancia de la punta de contacto a la pieza & \multicolumn{2}{|c|}{$25[\mathrm{~mm}]$} \\
\hline
\end{tabular}


Para llevar a cabo la ejecución de las soldaduras, se utilizó una fuente modelo inversal 300 multiprocesos de IMC, que contaba con la capacidad de seleccionar diferentes opciones de operación para el proceso GMAW, tales como tensión constante, corriente constante y corriente pulsada. En cuanto al posicionamiento y desplazamiento de las chapas metálicas, éstas fueron ajustadas a una placa sobre una base móvil prensada por medio de un brazo metálico con un controlador de velocidad, y en donde la antorcha se mantenía de manera fija, lo cual era esencial para la proyección del arco.

Con el fin de alcanzar uno de los objetivos planteados, se hizo un diseño donde se requería de la proyección del arco en abierto, para observar su comportamiento y longitud en el momento del proceso de soldadura A-GMAW, en comparación con la GMAW, de modo que para cumplir con este esquema se necesitó desarrollar un mecanismo de proyección y ampliación del arco, con la ayuda de una lente biconvexa, con la que se consiguió obtener una proyección del arco, con una ampliación de aproximadamente $12 \mathrm{X}$ del tamaño real de la longitud del arco, generando así un mayor control y análisis en la variación de dicho parámetro respecto a la transferencia metálica en curso [11]. Ver Figura 2.

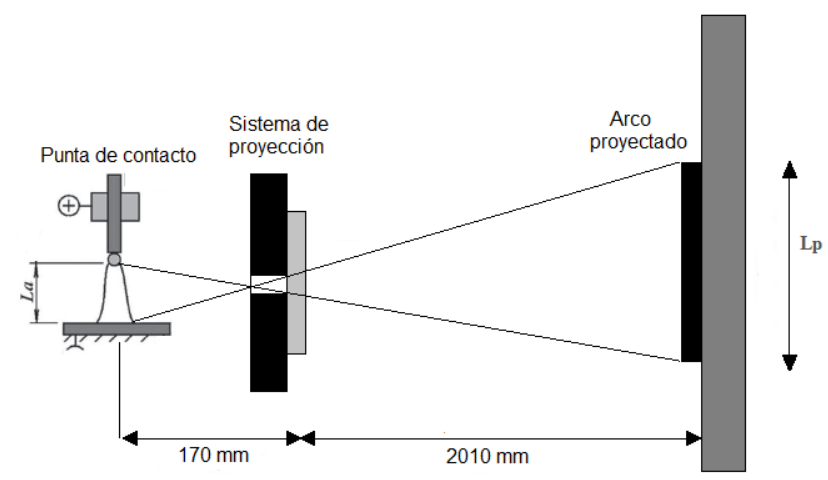

Figura 2. Diseño de la proyección de arco eléctrico. Fuente: Tomada y adaptada de Brito [11]. Lp = Longitud de la proyección.

Para la obtención de la longitud de arco deseada, se tuvo que ajustar la velocidad de alimentación, variando la tensión de referencia, a medida que se monitoreaba la longitud del arco en la pantalla de proyección, hasta llegar al valor de longitud de arco ya establecido como fijo $(6 \mathrm{~mm})$. Cuando la longitud del arco eléctrico encontrada se hacía igual al valor pre-establecido, se iniciaba la adquisición de las señales eléctricas de corriente, tensión de operación y velocidad de alimentación, con un sistema digital de adquisición de datos, con una tasa de $5000 \mathrm{~Hz}$. Luego estos datos serían procesados por medio del software PROGRAMA SINAL [12]. Para las soldaduras con flux, se mantuvo el mismo procedimiento, con la única diferencia de que el ajuste de la longitud del arco, se realizaba antes de que el arco eléctrico entrara en la zona con flux. Para esto, las chapas eran posicionadas de forma que el arco abriera en la zona sin flux, y una vez ya adentro de la capa de flux, la velocidad de alimentación no fue ajustada de nuevo, con el objetivo de observar las posibles variaciones en la longitud del arco eléctrico. Cabe aclarar que la realización de cada ensayo, tanto con y sin flux, se desarrolló en secuencia aleatoria y en duplicado.

Finalmente, para cumplir con el análisis morfológico de los cordones realizados, se necesitó cortar las chapas de forma transversal al cordón de soldadura, tanto en la región de soldadura convencional como en la A-GMAW, acompañado de toda la preparación metalográfica, para finalmente realizar un registro macrográfico con ayuda de una cámara profesional. Estos registros fotográficos fueron procesados en el software ImageJ.

\section{Resultados y Discusión}

\subsection{Adquisición de señales emitidas en las variables del proceso GMAW}

Para mayor facilidad en el estudio de estas señales, se ordenaron ascendentemente respecto con la corriente utilizada en cada operación y se obtuvo una relación para cada una de éstas, con las variables dependientes del proceso, y así se consiguió el valor de la media $(\bar{X})$, su desviación estándar (S), tiempo de corto-circuito (tcc) y factor de corto-circuito (Fcc). Este último indica el porcentaje de la duración en que el electrodo permanece haciendo contacto con el charco de fusión, durante un ciclo de la transferencia y se toma como un índice de estabilidad del proceso. Para la obtención de los valores de tcc y Fcc, se utilizaron las Ecuaciones 1 y 2, respectivamente. En la Figura 3 se hace una ilustración de las variables que comprenden la Ecuación $1[13,14]$.

$$
\begin{aligned}
& \mathrm{t}_{\mathrm{cc}}=\mathrm{T}-\mathrm{t}_{\mathrm{aa}} \\
& \mathrm{F}_{\mathrm{cc}}=\frac{\mathrm{t}_{\mathrm{cc}}}{\mathrm{T}} * 100
\end{aligned}
$$




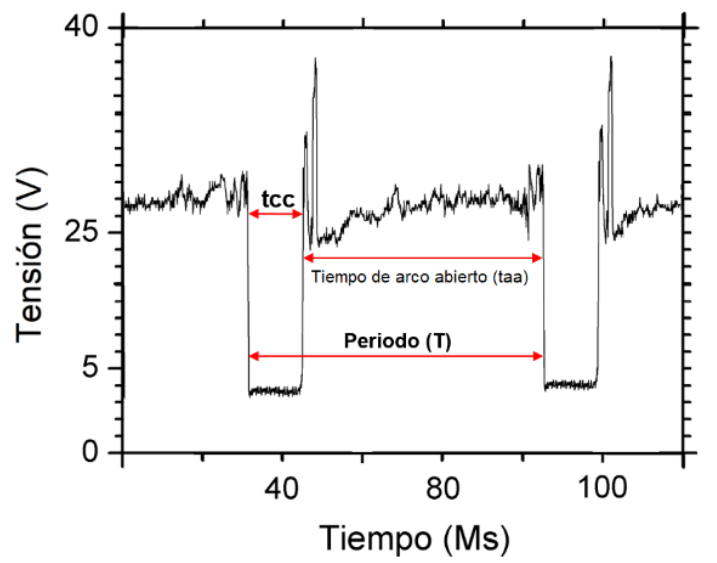

Figura 3. Oscilograma de corto-circuito. tcc = tiempo de corto-circuito. Adaptación propia.

En la Tabla 2, se muestran los datos obtenidos del programa SINAL, por medio de las señales adquiridas de las soldaduras del proceso GMAW convencional, emitidas en forma de oscilogramas por este programa. Después del análisis de los oscilogramas y de los valores obtenidos de esta tabla, se pudo ver que para el valor de 170 amperios [A], hubo una clara presencia de transferencia por corto circuito; esto no quiere decir que este proceso no se encuentre acompañado de metal transferido por vuelo libre, el cual tendría lugar entre un corto y el suceso del siguiente (arco en abierto). Observando el valor de Fcc obtenido (4,7\%) y comparándolo con el alcanzado en un proceso de corto-circuito realizado por Ferreira et al. [15], quien consiguió un valor de 8,6\% con el mismo material base utilizado en este trabajo, da cierto respaldo a lo planteado anteriormente, teniendo en cuenta que la distancia del tubo de contacto utilizada en este trabajo fue mucho mayor a la utilizada por Ferreira, lo que jugó un papel muy importante, ya que es mayor el desplazamiento que realizo el alambre electrodo, así como mayor la tensión del sistema, lo que puede terminar en un desprendimiento de las gotas antes de ocasionarse un corto. Por esto mismo, fue en éste valor de corriente donde se presentó la mayor reducción en la tensión promedio del arco, ya que, al presentarse corto circuito en el sistema, la longitud del arco disminuye hasta que el electrodo choca con la placa metálica, lo que generó una disminución significativa en el valor promedio de este potencial.

Para el valor de 180A, se evidenció una disminución en el Fcc, en comparación con el presentado en $170 \mathrm{~A}$, lo que indico un aumento en la transferencia metálica por vuelo libre, que se dedujo es de tipo globular. Siguiendo, con una corriente de 190A, se encontró un proceso gobernado por una transferencia de tipo globular con un Fcc muy cercano a cero, tal que, en el rango analizado de 10 segundos en promedio, el programa SINAL solo detectó 12 eventos que etiquetó como cortos, pero que se podrían asociar con un diámetro de gota muy grande y un desprendimiento de la misma aún muy lento, que deja como resultado un contacto con el charco de fusión antes de ésta desprenderse por completo del electrodo. Este valor se tomó como uno de los tres a utilizar en las soldaduras con el proceso A-GMAW, teniendo en cuenta que se buscaba un valor levemente por encima de condiciones con corto-circuito, uno en globular próximo a la región de transición (globular-spray), y uno de tipo spray, que fueron 190,210 y 230 A respectivamente.

Tabla 2. Valores de las señales del proceso GMAW convencional.

\begin{tabular}{|c|c|c|c|c|c|c|}
\hline \multirow{2}{*}{$\begin{array}{c}1 \\
\text { (A) }\end{array}$} & \multicolumn{2}{|c|}{ Tensión (V) } & \multicolumn{2}{|c|}{ Vel. Alimentación (m/min) } & \multirow{2}{*}{ Fcc (\%) } & \multirow{2}{*}{$\begin{array}{l}\text { Frecuencia de } \\
\text { Transferencia } \\
\text { (Hz) }\end{array}$} \\
\hline & $\overline{\mathbf{x}}$ & S & $\overline{\mathbf{x}}$ & S & & \\
\hline 170 & 25,3 & 4,9 & 4,2 & 0,20 & 4,7 & 11,7 \\
\hline 180 & 26,4 & 4,2 & 4,4 & 0,28 & 3,0 & 7,0 \\
\hline 190 & 28,2 & 1,9 & 4,8 & 0,16 & 0,4 & 1,5 \\
\hline 200 & 28,6 & 1,8 & 5,0 & 0,17 & - & - \\
\hline 210 & 28,0 & 0,8 & 5,4 & 0,08 & - & - \\
\hline 220 & 27,5 & 0,6 & 6,2 & 0,09 & - & - \\
\hline 230 & 27,6 & 0,4 & 6,5 & 0,07 & - & - \\
\hline
\end{tabular}

$\mathrm{A}=$ Amperios; Fcc = Factor de corto circuito; $\overline{\mathrm{X}}=$ Media; $\mathrm{S}=$ Desviación estándar.

Por medio de los oscilogramas, se pudo ver cómo con el aumento de la corriente, la tensión se va comportando cada vez más estable, esto debido al tipo de transferencia que se va presentando, pues a medida que el metal va dejando de transferirse por contacto con el pozo de fusión y comienza el desprendimiento de gotas, que con el incremento de la corriente se van 
haciendo cada vez más pequeñas y con una mayor velocidad de deposición, va generando que la longitud del arco se torne más constante, debido a que la punta del electrodo no alcanza a desplazarse hasta el charco de fusión, cuando ésta se ha fundido (aumento en la velocidad de fusión) y esto se refleja en una tensión más estable, que podemos comprobar con la disminución del desvió estándar (S) de la tensión, mostrado en el Tabla 2.

Para tener una mayor certeza de qué tipo de transferencia se estaba llevando a cabo en cada una de las corrientes, se analizaron varios aspectos durante y después del proceso; entre los analizados durante el proceso estuvieron la proyección del arco, con el que se podía ver qué tan estable se hacia la longitud del mismo, lo que servía de referencia; al igual que el sonido emitido por cada soldadura, que se asociaba a los predeterminados para cada tipo de transferencia. Otro método indirecto utilizado después del proceso, fue el análisis de los oscilogramas tanto estadísticamente (representado en las Tablas 2, 3 y 4), como por observación del mismo gráfico, para lo cual se tuvo en cuenta lo expuesto por Modenesi y Bracarense [4], quienes estiman que en la transferencia de tipo globular hay un desprendimiento de decenas de gotas/segundo; y para la de tipo spray, esta razón aumenta a centenas de gotas/segundo. Dichos desprendimientos de gotas se pueden evidenciar en el oscilograma de la Figura 4, con los picos de tensión (círculo rojo), que están acompañados de una leve caída de este parámetro, que estaría reflejando el crecimiento de la gota de forma longitudinal, antes de desprenderse del electrodo. Teniendo en cuenta un conteo de estos picos o caídas de tensión en la Figura 4, se podría decir que hubo una deposición de alrededor de 22 gotas/segundo en la soldadura del proceso GMAW convencional de $210 \mathrm{~A}$, catalogando a ésta como una transferencia de tipo globular. La homogeneidad de los picos o caídas de tensión, es un punto de referencia a tener en cuenta al momento de catalogar los modos de transferencia que gobiernan el proceso. Es por esto que, las soldaduras entre el rango de corrientes $>210 \mathrm{y}<230 \mathrm{~A}$, fueron clasificadas como de transición, por presentar una variación en la tendencia de las caídas de tensión y picos del oscilograma (menos homogeneidad en el oscilograma).

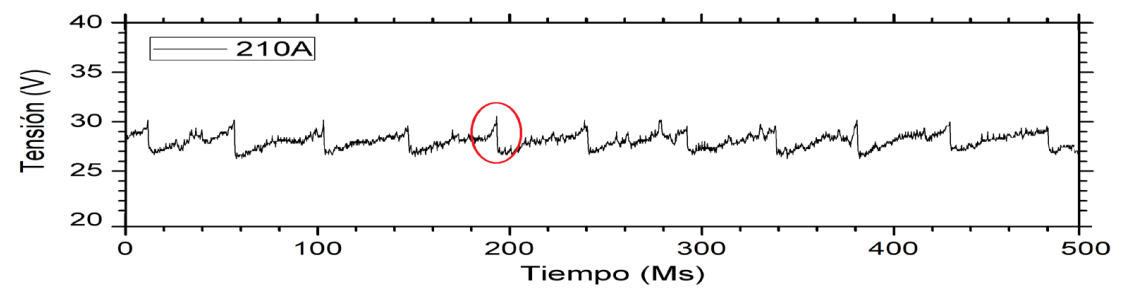

Figura 4. Oscilograma de tensión emitido por el programa SINAL, para 210A en GMAW convencional.

En las Tablas 3 y 4, se pueden ver los resultados obtenidos del proceso A-GMAW, para los tres valores de corriente escogidos, con flux de $\mathrm{SiO}_{2}$ y $\mathrm{CaCO}_{3}$, respectivamente. En ambas tablas se registraron los datos correspondientes a las señales antes de entrar en la zona con flux y dentro de ésta. De este modo se puede apreciar, que para las señales del valor de 190A sin flux, se presenta el mismo fenómeno ya descrito en el análisis anterior para este valor. Igualmente, estos valores se confirman por sus respectivos oscilogramas, con los que se elaboró la Figura 5, que hace una comparación del comportamiento de la tensión sin flux, respecto a la conducta con flux, para cada uno de los tres valores de corriente.

En los oscilogramas correspondientes al compuesto de $\mathrm{SiO}_{2}$ (Figura 5), se puede observar cómo después de que el arco entró en la zona con flux, se volvieron a presentar corto-circuitos en los tres valores de corriente, siendo el valor de $190 \mathrm{~A}$ el que mayor Fcc presenta, al igual que una frecuencia muy similar a la presentada por el valor de 170A sin flux, como lo muestra la Tabla 2 y 3 . Esto muestra, que el proceso ha vuelto a ser gobernado en gran parte por la transferencia de tipo corto-circuito.

Tabla 3. Valores de las señales del proceso A-GMAW con flux $\mathrm{SiO}_{2}$.

\begin{tabular}{|c|c|c|c|c|c|c|c|c|}
\hline \multirow{2}{*}{$\begin{array}{c}1 \\
\text { (A) }\end{array}$} & \multirow{2}{*}{$\begin{array}{l}\text { Con flux } \\
\text { (x) }\end{array}$} & \multicolumn{2}{|c|}{ Tensión (V) } & \multicolumn{2}{|c|}{$\begin{array}{l}\text { Vel. Alimentación } \\
\text { (m/min) }\end{array}$} & \multirow{2}{*}{$\begin{array}{l}\text { Longitud arco } \\
\qquad(\mathrm{mm})\end{array}$} & \multirow[t]{2}{*}{ Fcc (\%) } & \multirow{2}{*}{$\begin{array}{c}\text { Frecuencia de } \\
\text { Transferencia } \\
\text { (Hz) }\end{array}$} \\
\hline & & $\overline{\mathbf{X}}$ & S & $\overline{\mathbf{X}}$ & S & & & \\
\hline \multirow{2}{*}{190} & & 27,5 & 2,5 & 4,8 & 0,16 & 6,0 & 1,1 & 3,6 \\
\hline & $x$ & 27,1 & 6,5 & 5,0 & 0,29 & 5,3 & 7,4 & 11,5 \\
\hline \multirow{2}{*}{210} & & 28,2 & 0,8 & 5,7 & 0,13 & 6,0 & - & - \\
\hline & $x$ & 28,1 & 5,2 & 5,8 & 0,47 & 5,6 & 4,5 & 9,7 \\
\hline \multirow{2}{*}{230} & & 27,8 & 0,5 & 6,6 & 0,11 & 6,0 & - & - \\
\hline & $x$ & 27,7 & 5,2 & 6,9 & 0,45 & 5,7 & 5,0 & 14 \\
\hline
\end{tabular}

$\mathrm{A}=$ Amperios; Fcc = Factor de corto circuito; $\overline{\mathrm{X}}=$ Media; $\mathrm{S}=$ Desviación estándar; $\mathrm{x}=$ Com flux. 
Tabla 4. Valores de las señales del proceso A-GMAW con flux de $\mathrm{CaCO}_{3}$.

\begin{tabular}{|c|c|c|c|c|c|c|c|c|}
\hline \multirow{2}{*}{$\begin{array}{c}1 \\
(A)\end{array}$} & \multirow{2}{*}{$\begin{array}{l}\text { Con flux } \\
\text { (x) }\end{array}$} & \multicolumn{2}{|c|}{ Tensión (V) } & \multicolumn{2}{|c|}{$\begin{array}{l}\text { Vel. Alimentación } \\
\text { (m/min) }\end{array}$} & \multirow{2}{*}{$\begin{array}{l}\text { Longitud arco } \\
(\mathrm{mm})\end{array}$} & \multirow[t]{2}{*}{ Fcc (\%) } & \multirow{2}{*}{$\begin{array}{l}\text { Frecuencia de } \\
\text { Transferencia } \\
\text { (Hz) }\end{array}$} \\
\hline & & $\overline{\mathbf{x}}$ & $S$ & $\overline{\mathbf{x}}$ & $S$ & & & \\
\hline \multirow{2}{*}{190} & & 27,9 & 2,1 & 4,6 & 0,16 & 6,0 & 0,57 & 1,77 \\
\hline & $x$ & 28,0 & 1,9 & 4,4 & 0,25 & 9,6 & - & - \\
\hline \multirow{2}{*}{210} & & 27,8 & 0,9 & 5,5 & 0,10 & 6,0 & - & - \\
\hline & $x$ & 27,7 & 1,7 & 5,3 & 0,24 & 9,6 & - & - \\
\hline \multirow{2}{*}{230} & & 28,1 & 0,5 & 6,5 & 0,11 & 6,0 & - & - \\
\hline & $x$ & 28,1 & 0,9 & 6,2 & 0,18 & 10,7 & - & - \\
\hline
\end{tabular}

$\mathrm{A}=$ Amperios; Fcc = Factor de corto circuito; $\bar{X}=$ Media; $\mathrm{S}=$ Desviación estándar; $\mathrm{x}=$ Com flux.

A pesar de que los valores 210 y $230 \mathrm{~A}$, tienen Fcc similares a los de $170 \mathrm{~A}$ sin flux, y frecuencias de transición parecidas, en ocasiones mayores que las presentadas en los 190A con flux, no se podría afirmar que, en dichos procesos, se está presentando la misma estabilidad y gobernabilidad por parte del corto-circuito, debido a que estos aspectos se definen por medio del cálculo del desvió estándar (S) del periodo y el análisis visual de los oscilogramas.

En este caso, para la corriente de $230 \mathrm{~A}$ el periodo tiene una variación de $0,0973 \mathrm{~s}$, mayor que su propio periodo promedio $(T=0,0906 \mathrm{~s})$, en comparación con una variación de $0,0467 \mathrm{~s}$ presentado por los datos del valor de $190 \mathrm{~A}$ en donde el periodo fue de $T=0,0875 \mathrm{~s}$, que en otras palabras se traduce en mayor homogeneidad en los tiempos de sucesos de los cortos. Si se observa la Figura 5, se podrá ratificar como para los valores de 210 y $230 \mathrm{~A}$, se encuentran regiones más amplias donde la transferencia estuvo gobernada por los métodos de vuelo libre; así como se ve en algunos casos un tiempo de corto muy reducido, lo que hace pensar que se puede estar presentando el mismo fenómeno de la gota que toca el charco de fusión, instantes antes de desprenderse, teniendo en cuenta que la longitud de arco se hizo más pequeña; situación que podría respaldar esta hipótesis.
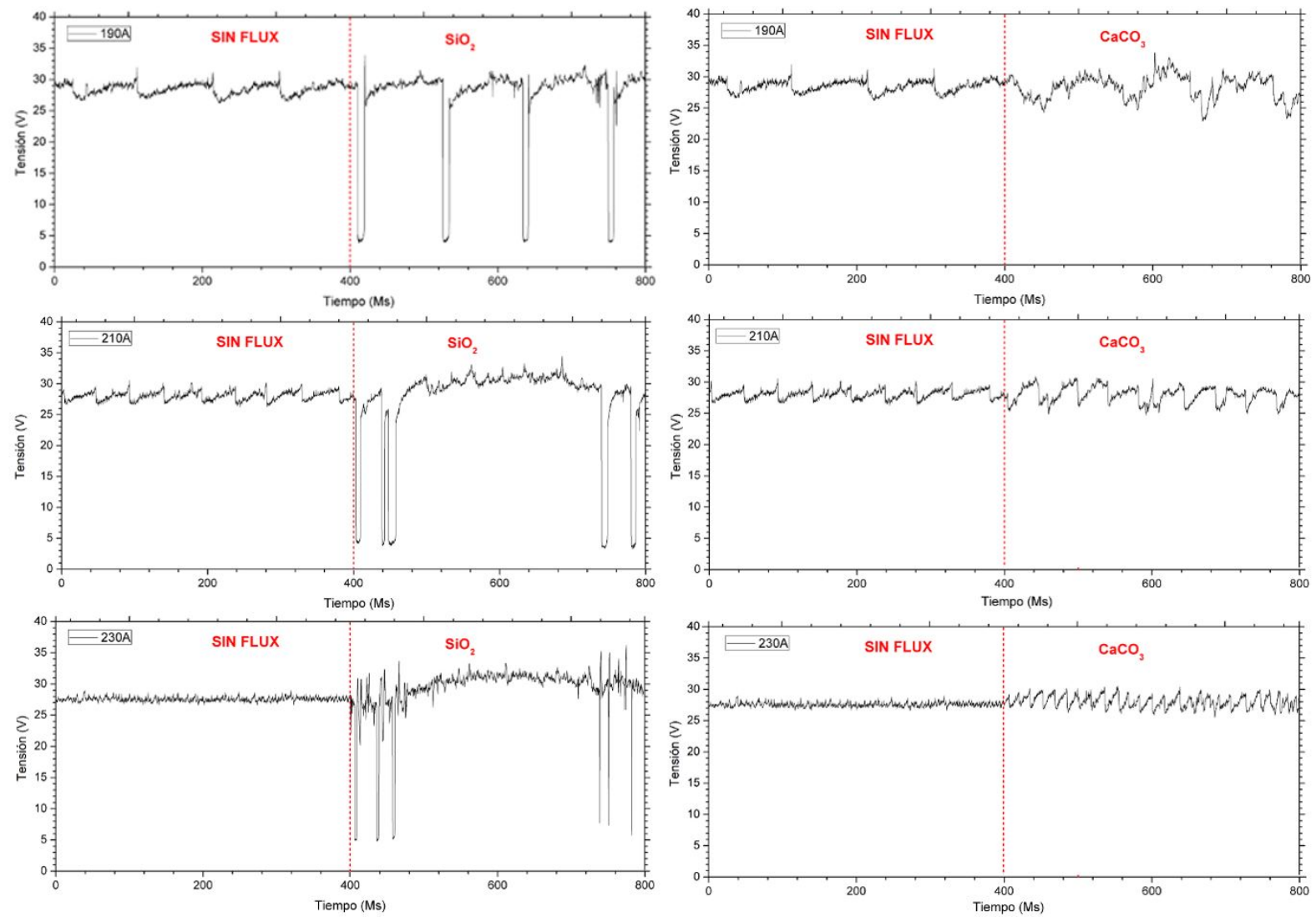

Figura 5. Oscilogramas comparativos del proceso GMAW convencional y el A-GMAW. 
Para el caso del $\mathrm{CaCO}_{3}$, ninguna de las soldaduras con el flux presentó algún tipo de corto; esto también puede estar relacionado con que el valor de la longitud del arco eléctrico presente aquí fue mucho mayor, lo que hace más difícil el acontecimiento de un corto-circuito como tal o de un choque de la gota antes de desprenderse del electrodo. Aún así, se observó una alteración en las señales de la tensión (Figura 5), lo que podría estar asociado con el incremento de $\mathrm{CO}_{2}$ al gas protector, proveniente de la descomposición del flux. Este aumento estaría originando una fuerza de repulsión mayor en la transferencia metálica, lo que ocasionaría una demora en el desprendimiento de la gota y por ende un aumento de su tamaño, generando cierto retraso en el método de transferencia en cuanto a los valores de corriente para el tipo spray $[13,16]$.

Teniendo en cuenta las Tablas 2, 3 y 4, se realizó un análisis comparativo de la velocidad de alimentación y de la longitud del arco eléctrico, como se muestra en las Figuras 6,7 y 8.

En la Figura 6, se puede apreciar de forma muy clara, cómo se presentó un incremento de la velocidad de alimentación a medida que se aumenta la corriente del proceso. Esto tendría una explicación, que consiste en que cuando se aumenta la corriente, la velocidad con la que se funde el electrodo se hace mayor, lo que obliga al sistema a suministrar más alambreelectrodo (aumentar la velocidad de alimentación a través de la tensión de referencia) para de este modo compensar el alambre fundido y poder mantener la longitud de arco $(6 \mathrm{~mm})$ constante.

Para poder entrar analizar comparativamente la velocidad de alimentación en el proceso GMAW convencional vs el A-GMAW, es conveniente primero ver lo sucedido con la longitud del arco en estos dos procesos. Para esto, se puede observar la Figura 7, donde tenemos como punto de referencia la longitud del arco en el proceso GMAW convencional, la cual se mantuvo constante $(6 \mathrm{~mm})$ después del ajuste realizado. Partiendo de allí, se observa cómo en presencia del $\mathrm{CaCO}_{3}$, la longitud del arco tuvo un incremento notable y aún más sobre el último valor de corriente, lo que intuitivamente hace pensar en principio, que hubo una disminución en la velocidad de alimentación. Por el contrario, en presencia del $\mathrm{SiO}_{2}$, el arco se tornó más pequeño longitudinalmente, haciéndose más notable en el valor de 190A, y esto haría creer que hubo una mayor velocidad de alimentación, en comparación con la velocidad de fundición del alambre. Sin embargo, dichas disminuciones en la velocidad tendrían que ser mínimas, pues en el proceso no se modificó este parámetro (tensión de referencia), lo que haría que el sistema intente siempre enviar la misma cantidad de alambre por unidad de tiempo. Esto último es verificado por medio de la Figura 8 , que muestra las mínimas variaciones, respecto al ajuste hecho en la velocidad de alimentación para cada operación.

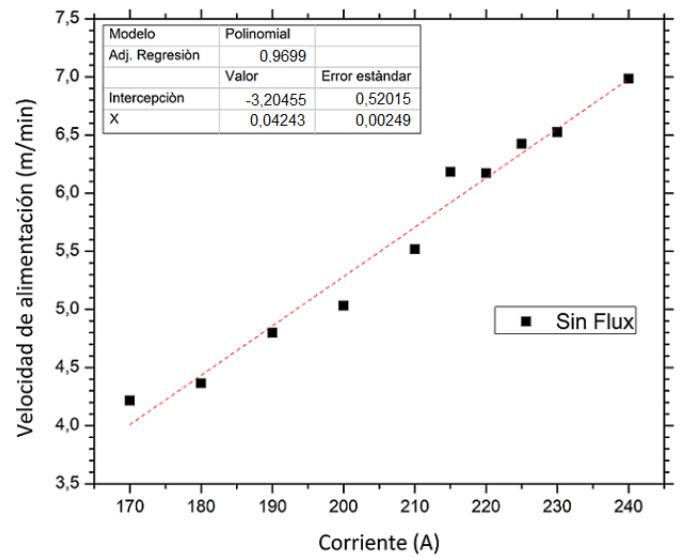

Figura 6. Variación de la velocidad de alimentación del electrodo en el proceso GMAW convencional.

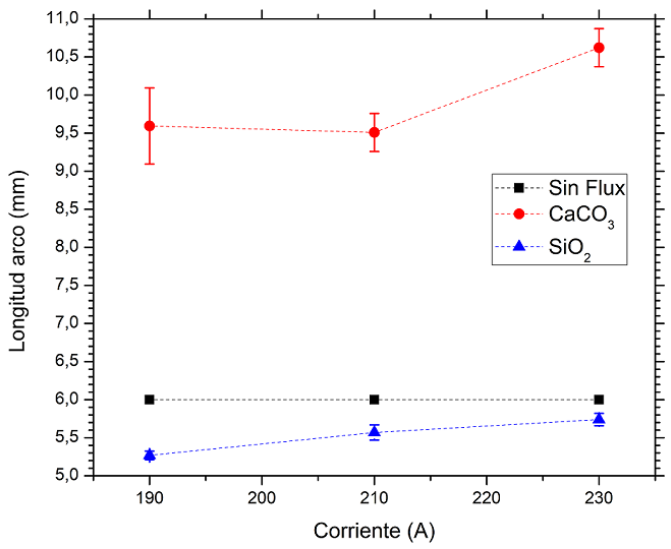

Figura 7. Comparación de la longitud de arco eléctrico de los procesos GMAW convencional y A-GMAW. 


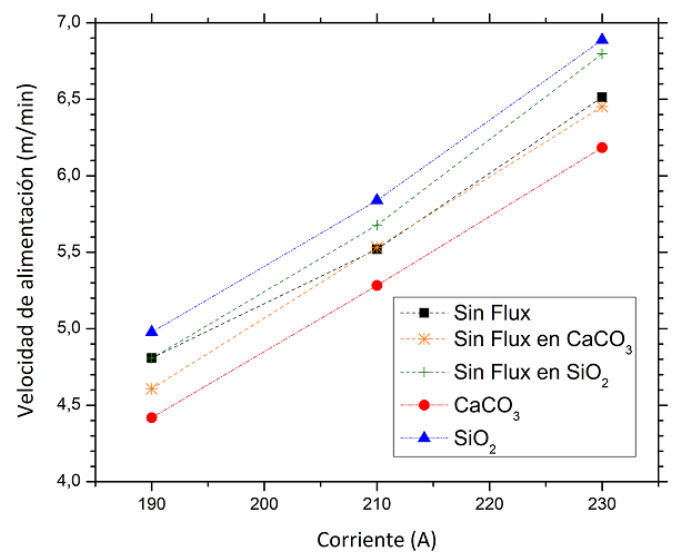

Figura 8. Comparación de la variación de velocidad de los procesos GMAW convencional y A-GMAW.

De esta manera se puede suponer entonces, que las variaciones se presentan en la tasa de fusión del electrodo, debido a las modificaciones que se estarían presentando en el gas de protección, por la descomposición de los active flux y la mezcla de estos con el gas protector.

Para el caso específico de $\mathrm{CaCO}_{3}$, la atmósfera protectora puede estar sufriendo un aumento del tenor de $\mathrm{CO}_{2}$, derivado de la descomposición térmica del flux de carbonato de calcio $\left(\mathrm{CaCO}_{3} \rightarrow \mathrm{CaO}+\mathrm{CO}_{2}\right)$, que aunque llevaría a un incremento mínimo en el porcentaje de dióxido de carbono en la composición del gas protector ( $\approx 1 \%)$ [16], éste podría estar influenciando en el perfil del arco. Lo anterior, debido a que el $\mathrm{CO}_{2}$ posea alta energía de disociación/ionización y elevada conductividad térmica, lo que estaría relacionado con el incremento de la longitud del arco, ya que ocasionaría una mayor tasa de fusión del electrodo y por ende, una reducción en el Stick-out (alambre-electrodo, sobresaliente de tubo de contacto) Ver Figura 7.

\subsection{Análisis de los efectos de los active flux, en la morfología del cordón de soldadura}

En primer lugar, se realizó una comparación visual de la forma y tamaño de los cordones de soldaduras. Donde se encontró que el cordón en la zona con flux $\left(\mathrm{SiO}_{2}\right)$ se tornó menos homogéneo en su ancho; igualmente sucedió con el flux de CaCO3. Para dicho efecto se tendría varias hipótesis, basadas en la homogeneidad y continuidad del flux en el área. En primer lugar, se piensa que pudo no haber un esparcimiento homogéneo del flux en la zona aplicada, lo cual conllevaría a una variación en su densidad, por lo que genera una diferencia en el efecto del mismo. De igual forma podría haber sucedido que dicho flux hubiese sido retirado de la zona por medio de las fuerzas de soplo del gas protector o del plasma del arco eléctrico, teniendo en cuenta que para la deposición del flux no se utilizó ningún adherente, lo que haría que dicho flux, pudiera ser removido con facilidad. Por último, se sabe que por las altas temperaturas que envuelven el proceso, ocurre una evaporación del flux, lo que podría estar sucediendo mucho antes de que el charco de fusión tocara el flux, disminuyendo de este modo la densidad del mismo.

Para poder hacer una mejor comparación del ancho de los cordones, se tomaron mediciones por medio de un calibrador y se analizaron las macrografías de los perfiles (Figura 9), de donde se derivó la Figura 10, que muestra como la constricción del ancho del cordón fue de cierto modo muy similar para las soldaduras con flux, pero muy angostas comparadas con las realizadas sin flux.

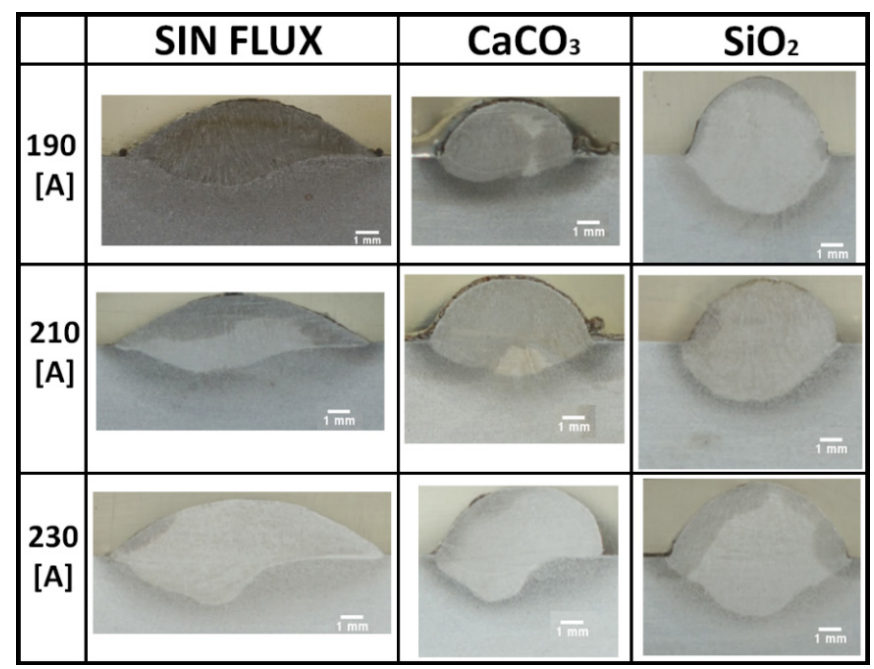

Figura 9. Macrografía de los perfiles de los cordones de soldadura. 


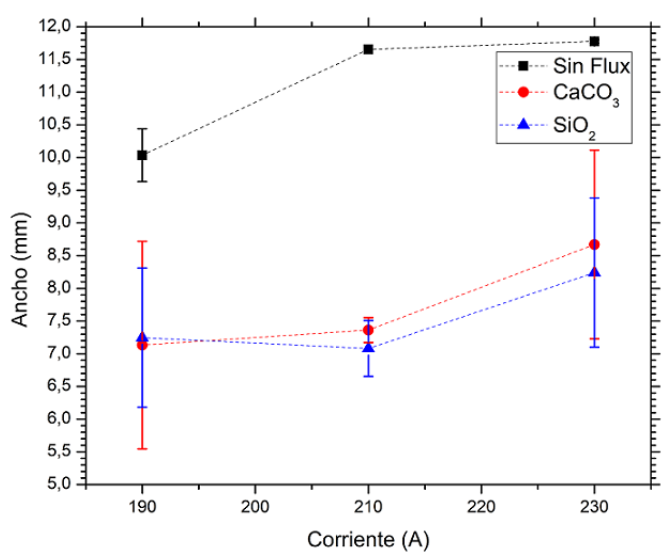

Figura 10. Comparación de la variación del ancho del cordón respecto al proceso GMAW convencional y A-GMAW.

De igual forma, también se analizó el área del perfil de la Zona Fundida (ZF), para lo cual se utilizó la Ecuación 3, donde G es el área, S la velocidad de alimentación, d el diámetro del electrodo y $\mathrm{R}$ la velocidad de soldadura [17]. Se observa que la ecuación se encuentra únicamente regida por la velocidad de alimentación (S), debido a que todas las otras variables se mantendrán constantes y por ende, se puede predecir el resultado de estos valores, observando las Figuras 6 y 8 , ya que tomarían el mismo comportamiento. Se puede decir entonces, que hubo un aumento proporcional de la deposición de material, respecto a la velocidad de alimentación, siendo entonces, el $\mathrm{SiO}_{2}$ el que mayor área presentó y el $\mathrm{CaCO}_{3}$ el de menor.

$\mathrm{G}=\frac{\mathrm{S} \pi \mathrm{d}^{2}}{\mathrm{R} 4}$

De las macrografías obtenidas de los cordones (Figura 9), se analizaron otros aspectos que definen su morfología, como lo es la penetración del material depositado que se representó por medio de un gráfico comparativo como se observa en la Figura 11. En dicha figura se aprecia como la penetración conseguida con el flux $\mathrm{SiO}_{2}$ fue mucho mayor que la adquirida sin ningún tipo de flux. Mientras que con el compuesto de $\mathrm{CaCO}_{3}$, éste valor se hizo en promedio menor que el de las soldaduras de referencia, a excepción del valor obtenido en la corriente de $210 \mathrm{~A}$ donde la penetración fue 0,15 mm mayor que la de referencia.

Estas variaciones de profundidad y forma del perfil son igualmente asociadas con los usos de los gases de protección por muchos autores $[4,13,16,17]$. Es por eso, que para el caso del $\mathrm{CaCO}_{3}$ que se supone alteró esta variante, generando una mayor longitud de arco, que acabo en un desprendimiento de la gota desde una distancia mayor y por ende en una pérdida de calor en el viaje de la misma, de tal manera que, al llegar a la piscina de metal, se manifestaría en una menor penetración. De igual forma como se planteó en un análisis anterior para este flux, el aumento de las fuerzas de repulsión estaría ocasionando un crecimiento de la gota y esto retardó la deposición de la misma, viéndose reflejada tanto en el área del perfil, como en la penetración, pues al hacerse mayor la gota y más lenta la deposición, tendría un menor impacto en el charco que cuando se envían en forma de spray.

Ahora, en cuanto al flux de $\mathrm{SiO}_{2}$, que también presentaría cierta descomposición de oxígeno hacia la atmósfera protectora, al igual que para el charco de fusión, lo que estaría generando en éste último, el llamado efecto Marangoni, que prevalece en los flux a base de oxígeno, justificando así, los grandes valores en la penetración $[8,18]$.

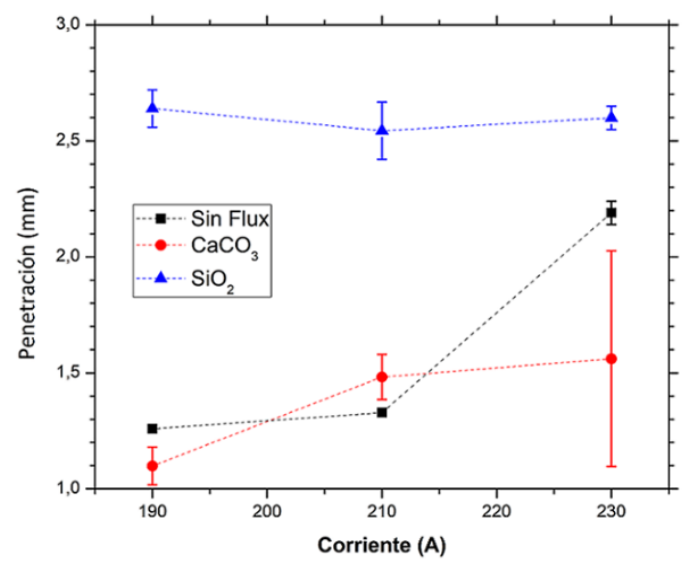

Figura 11. Comparación de la penetración de las soldaduras en el proceso GMAW convencional y A-GMAW. 


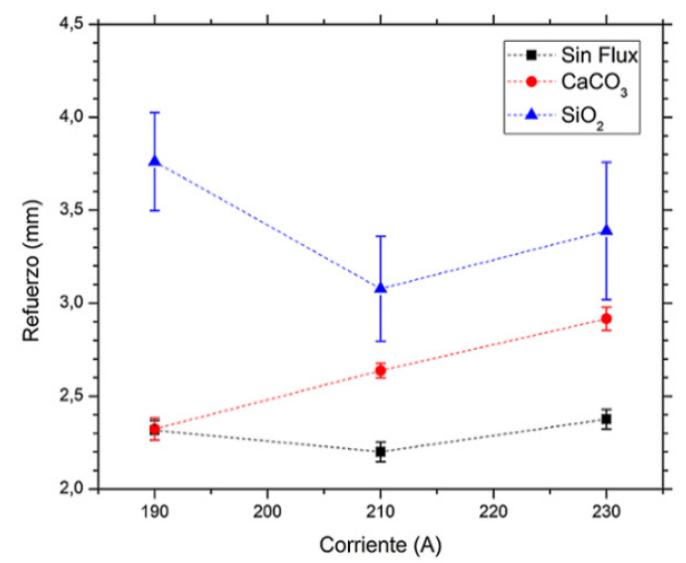

Figura 12. Comparación del refuerzo de la soldadura en el proceso GMAW convencional y A-GMAW.

Por último, en la Figura 12 se observa que el elevado refuerzo en el cordón de $\mathrm{SiO}_{2}$ está asociado de igual forma con la mayor deposición de metal y en el mismo efecto Marangoni, que no permitió que el metal se expandiera a lo ancho de la placa. Por otro lado, el $\mathrm{CaCO}_{3}$, al tener una penetración tan baja, terminó generando un refuerzo elevado con todo aquel material que no penetró en el metal.

\subsection{Análisis visual del arco eléctrico}

La variación de la longitud del arco eléctrico, no fue el único aspecto analizado por medio de la proyección del arco. Com este sistema también se encontró una variación en la constricción del arco como se planteó anteriormente, así como un cambio en la iluminación. Es así como al grabar lo proyectado en la pantalla, se pudo analizar a más baja velocidad lo sucedido, y se capturó la Figura 13, donde se puede apreciar el aumento de la longitud del arco para ese proceso específico, así como una mayor iluminación del arco, la cual se evidenció en todas las soldaduras con el proceso A-GMAW, y fue siempre mayor en el centro del arco, siendo más notorio con el flux de $\mathrm{CaCO}_{3}$. Otros autores $[13,16]$ presenciaron un tipo de deflexión del arco al entrar en la zona con flux, al hacer un proyección y análisis similar a éste, cosa que no se logra observar desde estas capturas. Por otro lado tampoco fue posible comprobar el efecto de constricción del arco, debido al aumento en la luminosidad. Sería necesario una filmación de alta velocidad para poder asegurar la existencia o no de dicho efecto.

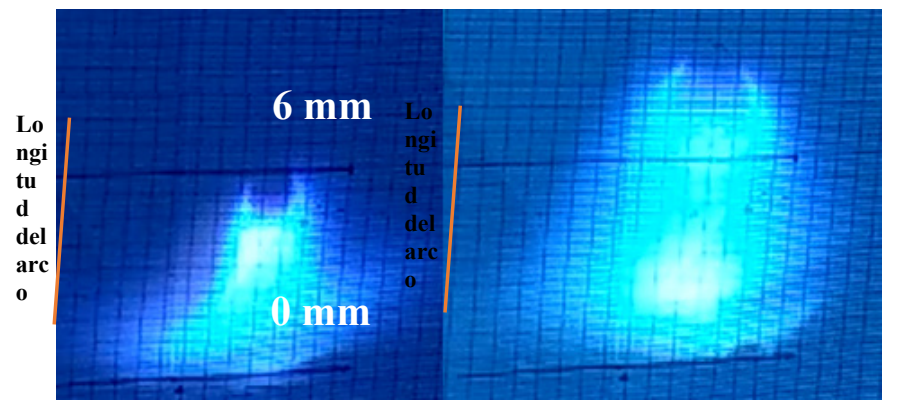

Figura 13. Captura del arco eléctrico proyectado en el proceso GMAW convencional y A-GMAW con $\mathrm{CaCO}_{3}$ a una corriente de $190 \mathrm{~A}$.

Los flux utilizados son formados por elementos fácilmente ionizables. Estos serían indicios de la presencia de vapores metálicos en el arco eléctrico, lo que llevaría a un aumento de la luminosidad. Para el caso concreto del $\mathrm{CaCO}_{3} \mathrm{Captado}$ en la Figura 13, se le adjudicaría dicho aumento de la iluminación a los cationes de calcio desprendidos del flux, los cuales estarían favoreciendo la emisión de radiación del arco $[13,16,19]$.

\section{Conclusiones}

Del presente trabajo acerca de los efectos ocasionados por los active flux de $\mathrm{SiO}_{2}$ y $\mathrm{CaCO}_{3}$, sobre soldaduras $\mathrm{GMAW}$ en un acero AISI/SAE 1020, se concluyó que:

- Al realizar el análisis de los datos obtenidos del programa SINAL, por medio de oscilogramas, se pudo concluir que tanto el $\mathrm{SiO}_{2}$ como el $\mathrm{CaCO}_{3}$ ocasionaron una variación en el método de transferencia metálica, generando alteraciones en la tensión del proceso; 
- Se observaron transferencias de metal por medio de corto-circuito con la utilización de $\mathrm{SiO}_{2}$; por el contrario, al implementarse el flux de $\mathrm{CaCO} 3$ se desaparecieron los corto-circuitos ocasionales presentes en los valores de corriente de 190A, en el proceso convencional;

- Se presentó un incremento de la velocidad de alimentación al tratar de mantener constante la longitud del arco, mientras se aumentaba la corriente en el proceso GMAW convencional. Este incremento se mantuvo sin variaciones notables en el proceso A-GMAW ya que no se volvió a regular la longitud del arco;

- Se asociaron las desviaciones de la longitud del arco, a efectos ocasionados con la variación de la composición de los gases protectores, por causas de una descomposición de los flux, concluyendo de este modo, que los compuestos de $\mathrm{SiO}_{2}$ y $\mathrm{CaCO}_{3}$ afectan la atmósfera protectora;

- Con la aplicación de los flux, se presentaron variaciones significativas en las características geométricas del cordón, como penetración, ancho y refuerzo, al ser comparadas con las obtenidas en el proceso GMAW convencional;

- Con la utilización del flux de $\mathrm{SiO}_{2}$, se obtuvo una mayor penetración respecto al proceso convencional, y con el flux de $\mathrm{CaCO}_{3}$ se consiguieron resultados complemente inversos;

- El ancho del cordón se mostró más angosto para ambos flux en comparación con el proceso convencional y se observó una alteración en el mismo, que se asoció a unas diferencias en la densidad del flux en la zona esparcida.

\section{Referencias}

[1] Bracarense AQ. Gas metal arc welding 2.2. Osasco: Infosolda; 2003.

[2] Scotti A, Ponomarev V, Lucas W. Interchangeable metal transfer phenomenon in GMA welding: features, mechanisms, classification. Journal of Materials Processing Technology. 2014;214(11):2488-2496. http://dx.doi.org/10.1016/j.jmatprotec.2014.05.022.

[3] Scotti A, Ponomarev V, Lucas W. A scientific application oriented classification for metal transfer modes in GMA. Journal of Materials Processing Technology. 2012;212(6):1406-1413. http://dx.doi.org/10.1016/j.jmatprotec.2012.01.021.

[4] Modenesi PJ, Bracarense AQ. Introdução à física do arco elétrico e sua aplicação na soldagem dos metais. Belo Horizonte: Universidade Federal Minas Gerais; 2012.

[5] Costa TF, Benedetti E Fo, Arevalo HDH, Vilarinho LO. Avaliação de processos MIG/MAG curto-circuito convencional e controlado para a soldagem de dutos de aço carbono em passe único. Soldagem e Inspeção. 2012;17(4):356-368. http://dx.doi.org/10.1590/S010492242012000400010 .

[6] Huang H-Y. Effects of activating flux on the welded joint characteristics in gas metal arc welding. Materials \& Design. 2010;31(5):24882495. http://dx.doi.org/10.1016/j.matdes.2009.11.043.

[7] Modenesi PJ, Apolinário ER, Pereira IM. TIG welding with single-component fluxes. Journal of Materials Processing Technology. 2000;99(1-3):260-265. http://dx.doi.org/10.1016/S0924-0136(99)00435-5.

[8] Modenesi PJ. A química da formação do cordão na soldagem TIG. Soldagem e Inspeção. 2013;18(3):287-300. http://dx.doi.org/10.1590/S0104-92242013000300011.

[9] Ferreira JR No. Estudo do efeito de fluxos em aspectos operacionais da soldagem MIG/MAG [dissertação de mestrado]. Belo Horizonte: Universidade Federal Minas Gerais; 2017.

[10] Colen P No. Efeito da concentração do fluxo na soldagem ATIG de aço inoxidável austenítico [dissertação de mestrado]. Belo Horizonte: Universidade Federal Minas Gerais; 2012.

[11] Brito L. Análise da influência dos fluxos $\mathrm{SiO} 2$ e $\mathrm{CaCO} 3$ sobre a geometria do cordão em soldagem A-GMAW [dissertação de pregrado]. Belo Horizonte: Universidade Federal Minas Gerais; 2017.

[12] Modenesi PJ. Manual Programa Sinal. Belo Horizonte: Laboratório de Robótica, Soldagem e Simulação, Universidade Federal Minas Gerais; 2017.

[13] Batista LA. Soldagem MIG/MAG com fluxo ativo do aço inoxidável austenítico AISI 304L [dissertação de mestrado]. Belo Horizonte: Universidade Federal Minas Gerais; 2018.

[14] Modenesi PJ, Costa MCMS, Santana IJ, Berganholi JPP. Estudo de alguns parâmetros básicos da soldagem GMAW com transferência por curto-circuito. Soldagem e Inspeção. 2011;16(1):12-21. http://dx.doi.org/10.1590/S0104-92242011000100003.

[15] Ferreira JR No, Lopes PB, Apolinário ER, Modenesi PJ. Uma avaliação da soldagem MIG/MAG com fluxos. In: Anais do XLII Congresso Nacional de Soldagem (CONSOLDA); 2016; Belo Horizonte. São Paulo: Associação Brasileira de Soldagem; 2016.

[16] Batista LA, Brito L, Madariaga CH, Azevedo M, Modenesi PJ. Efeito do uso de CaCO3 como fluxo ativo no modo de transferência metálica. In: Anais do XLIII Congresso Nacional de Soldagem (CONSOLDA); 2017; Joinville. São Paulo: Associação Brasileira de Soldagem; 2017.

[17] ASM International. Welding brazing and soldering. Materials Park; 1993. (Metals Handbook; 6). 
[18] Patel KR, Choudhary AB, Patel TM. Parametric optimization of gas metal arc welding process using activated flux on EN 8 BY Taguchi method: a review. In: Proceedings of the International Conference on Challenges in It, Engineering and Technology (ICCIET); 2014; Phuket. Thailand: Mechanical \& Automobile Engineering; 2014.

[19] Murphy AB. The effects of metal vapour in arc welding. In: Proceedings of the 19th Symposium on Physics of Switching Arc; 2011; Brno, Czech Republic. Brno: Brno University of Technology; 2011. 